\title{
Literaturbericht
}

Hannes Adomeit*

\section{Russland und China - auf dem Weg zur strategischen Partnerschaft?}

https://doi.org/10.1515/sirius-2018-2008

\section{Einleitung}

Nach der Annexion der Krim, dem Ausbruch des Konflikts in der Ukraine und der Verhängung westlicher Sanktionen gegen Russland im Jahr 2014 verkündete Russland lautstark seine „Wende nach Osten“, eine Neuausrichtung seiner Außen- und Wirtschaftspolitik weg vom Westen. Die hier vorgestellten Beiträge befassen sich mit den Perspektiven der Kooperation mit der Volksrepublik China.

\section{Die Bedeutung von „strategischen Partnerschaften“}

Für Russland soll eine engere Partnerschaft mit China eine lebensfähige Alternative zu den Beziehungen zum Westen bieten. Michal Makocki, Senior Visiting Fellow am Mercator Institut für Chinastudien (MERICS) und Senior Associate Analyst am Institut für Sicherheitsstudien der Europäischen Union (EUISS), sowie Nicu Popescu, Senior Analyst ebenfalls am EUISS, haben sich in ihrem 51 Seiten umfassenden Chaillot Paper mit dieser Partnerschaft befasst.

Die Autoren gehen eingangs auf den Begriff der „strategischen Partnerschaft" ein und stellen fest, dass China derartige Partnerschaften mit über 50 Ländern und Organisationen geschlossen hat, die von der EU als Ganzes bis nach Angola, Belarus und Venezuela reichen. Um guten Willen zu zeigen, akzeptiere Peking die meisten Anfragen für solche Partnerschaften, aber nicht alle hätten das gleiche Gewicht. Es gebe etwa ein halbes Dutzend Kategorien unterschiedlicher Intensität, Tiefe und Bedeutung. Von diesen sei die höchste Form die von China so

*Kontakt: Dr. Hannes Adomeit: Senior Fellow, Institut für Sicherheitspolitik an der Universität Kiel, E-Mail: hannes.adomeit@t-online.de benannte „umfassende strategische Partnerschaft der Koordinierung“, die Russland vorbehalten sei.

Russland werde aus vielerlei Gründen hervorgehoben. So gebe es zunehmend Parallelen, wie die Eliten der beiden Länder die Welt sehen. In ihrer Weltanschauung würden die Vereinigten Staaten als schwindende Supermacht wahrgenommen, China und Russland hingegen als aufstrebende Mächte, denen größerer Status und Einfluss in der internationalen Politik gebühre. Es gebe aber noch weitere Gemeinsamkeiten. Dazu gehörten die wahrgenommene beziehungsweise vom Kreml so dargestellte westliche Feindseligkeit sowie die Besorgnis vor militärischem und wirtschaftlichem Containment. China fühle sich im Südchinesischen Meer unter Druck gesetzt, Russland von der NATO in der Ostsee. China sehe sich dem Aufbau von anti-ballistischen Raketensystemen in Südkorea und Japan ausgesetzt, Russland in Rumänien und Polen. Und während sich Moskau einer nach Osten expandierenden NATO gegenübersehe, schaue Peking auf eine verstärkte Präsenz der USA in Asien und auf Japans Verfassungsreform, die seine militärische Handlungsfreiheit und Macht stärken solle.

Als starke Triebkraft für Chinas Hinwendung zu Russland glauben die Autoren unter Hinweis auf Yan Xuetong, einen der bedeutendsten und einflussreichsten strategischen Denker Chinas, „strategischen Pessimismus“ zu erkennen. Dieser gehe auf die Wahrnehmung einer im Entstehen begriffenen bipolaren Welt zurück, die durch einen scharfen Gegensatz zwischen China und den USA gekennzeichnet sei. China brauche infolgedessen Verbündete und sehe keine bessere Möglichkeit als den Ausbau der Beziehungen zu Russland. Die Rivalität mit den USA müsse jedoch nicht zwangsläufig zum Krieg führen. Yan Xuetong argumentiert: „Wenn die Konfrontation zwischen den USA und der Sowjetunion wie ein Boxkampf mit Gewalt eine reguläre Erscheinung war, wird der Wettbewerb zwischen den USA und China eher wie ein Fußballspiel sein, bei dem Gliedmaßen gelegentlich kollidieren, aber Gewalt nicht die Hauptform der Rivalität ist. Das Verhältnis USA-China wird in erster Linie ein Team-Wettbewerb sein, dessen Ziel es ist, die Unterstützung anderer Länder zu gewinnen. Daher braucht das Team China 
eine ruhige nördliche Grenze, die es ihm ermöglicht, sich auf Herausforderungen im Süden und Osten zu konzentrieren.“

\section{Asymmetrien im Verhältnis zwischen Russland und China}

Marcin Kaczmarski vom Finnischen Institut für Internationale Angelegenheiten sieht das ähnlich. Er geht davon aus, dass sich die russisch-chinesischen Beziehungen im vergangenen Jahrzehnt unter den Bedingungen einer doppelten Asymmetrie entwickelt haben. „Erstens hat sich die Kluft zwischen den materiellen Fähigkeiten beider Staaten - gemessen am BIP, Handel, Investitionen und Militärhaushalten - stetig zugunsten Chinas vergrößert. Zweitens brauchte Russland Chinas Unterstützung mehr als China Russland benötigte. " Hier ist ein gewisser Widerspruch festzustellen, denn im Titel der Studie ist davon die Rede, dass sich Asymmetrien im beiderseitigen Verhältnis abgeschwächt hätten. Dafür gibt der Verfasser aber keinen Beleg.

Die Asymmetrie in der Sicherheitspolitik, so Kaczmarski, gehe auf die Zeit zurück, in der China ein relativ gutes Verhältnis zu den USA unter Präsident Barack Obama pflegen und von der offenen Weltordnung habe profitieren können. China habe es daher vermieden, Partei zu ergreifen, und Russland nicht bei seiner revisionistischen Politik gegenüber dem Westen unterstützt. Die unter Präsident Donald Trump zu beobachtende Änderung des USamerikanischen Ansatzes gegenüber China habe aber die politischen Asymmetrien verringert.

Diese Wertung deckt sich in gewisser Weise mit dem von Yan Xuetong festgestellten „strategischen Pessimismus“. Dennoch stellt sich die Frage, wie weit sich die sicherheitspolitischen Asymmetrien in den russisch-chinesischen Beziehungen tatsächlich abgeschwächt haben und ob sich Russland in seiner „Partnerschaft“ mit China nicht nur ökonomisch, sondern auch politisch in eine Juniorenrolle manövriert hat. $\mathrm{Zu}$ diesem Schluss kommen jedenfalls Makocki und Popescu. Trotz des optimistischen Tonfalles der offiziellen Äußerungen werde die Beziehung aus mehreren fundamentalen Gründen nicht der Rhetorik gerecht. Jede Seite hege unterschiedliche Erwartungen und habe unterschiedliche Interessen. Die Synergien zwischen den Volkswirtschaften beider Länder seien begrenzt. Die Komplementaritäten zwischen Russlands Rohstoffreichtum und Chinas energieintensivem Wachstum würden ebenfalls übertrieben. Die wirtschaftlichen Gravitationszentren dieser beiden Nachbarn lägen geografisch sehr weit voneinander entfernt, zudem seien die sich widersprechenden geopolitischen Interessen insbesondere in Zentralasien ausgeprägt. Russland nehme dieses Gebiet als seinen eigenen Hinterhof wahr, aber China umwerbe es im Rahmen seiner ehrgeizigen „Belt and Road“-Initiative mit einem Versprechen von Investitionen. Insgesamt sei die Herstellung eines Bündnisses wie auch einer tiefgreifenden strategischen Ausrichtung „nahezu unmöglich“. Diese Situation werde von beiden Seiten höflich gehandhabt, aber in Wahrheit sei der Kampf hart - „besonders für Russland“.

\section{Rivalität und Kooperation}

Ähnlich urteilen auch Paul Stronski und Nicole Ng vom Carnegie Endowment for International Peace in Washington, D.C. Die Autoren untersuchen die Mischung aus Rivalität und Kooperation im russisch-chinesischem Verhältnis vor allem in Zentralasien, Russlands Fernen Osten und der Arktis und kommen dabei zu folgenden Ergebnissen:

- In Zentralasien habe China sein sicherheitspolitisches und wirtschaftliches Engagement erhöht. Es entwickle sich dort zu einem einflussreichen Akteur. Während die Aussichten für die Verwirklichung der chinesischen „Belt and Road“-Initiative gut seien, stehe es für die von Russland initiierte Eurasische Wirtschaftsunion eher schlecht.

- Russland betrachte die wirtschaftliche Entwicklung seines Fernost-Gebiets als große strategische Aufgabe. Um diese zu erfüllen, seien chinesische Investitionen unerlässlich. Diese seien aber nicht so umfangreich, wie es die Interessen Russlands erforderten. Zudem nutze China oft seinen wirtschaftlichen Einfluss, um günstige Handelsbedingungen zu erreichen.

- In der Arktis brauche Russland China, um seine Ziele für die Infrastrukturentwicklung und die Rohstoffgewinnung zu verwirklichen. Umgekehrt sei China bestrebt, sein wirtschaftliches Potenzial in der Arktis einzusetzen und durch die Partnerschaft mit Russland seine technologische Leistungsfähigkeit in Schlüsselprojekten zu verbessern. Doch dies stelle Russland vor neue Probleme, da es seine Souveränität in der Region voll und ganz bewahren wolle.

Insgesamt habe China die Oberhand in den Beziehungen, die Machtasymmetrie werde auf Kosten Russlands weiter wachsen. China werde die Weichen für die weitere Entwicklung stellen, worauf Russland nur reagieren könne. 
Dies beinhalte allerdings kaum Vorteile für den Westen. Das der Partnerschaft innewohnende Bemühen, das internationale System zum Vorteil der beiden Länder zu verändern, stelle eine große Herausforderung für den Westen dar. Die westliche Politik müsse sich auf die sowohl von Russland als auch China verfolgte, zunehmend aktivistische Außenpolitik einstellen.

\section{Gemeinsamkeiten unter autoritären Regimen?}

US-amerikanischen Forschern zufolge entsteht für China politischer Handlungsspielraum aus einer neuen autoritären „sharp power“. Paul J. Kohlenberg und Nadine Godehardt, beide Forschungsgruppe Asien der Stiftung Wissenschaft und Politik (SWP), teilen diese Auffassung nicht. Sie sehen die internationale Erfolgsgeschichte Chinas eher in der konsequenten Durchsetzung von „connectivity power“. Darunter verstehen sie die von Präsident Xi Jinping eingeleitete, global ausgerichtete Politik von Infrastrukturinvestitionen und internationaler Kreditvergabe, die Ausweitung der Kooperation mit Institutionen in Forschung, Finanzen und Politik, den Erwerb internationaler Medienhäuser und die Verbreitung eigener technischer und regulativer Standards. Im Einklang mit der (wenn auch nicht ausdrücklich genannten) Betrachtung Deutschlands als „Zivilmacht“ kommen sie zu dem Schluss, dass sich aus der chinesischen Konnektivitätspolitik ,weit über den Infrastrukturbereich hinaus politische Handlungsoptionen für Deutschland und die EU“ eröffnen.

\section{Anspruch und Realität}

Praktisch alle Autoren sind sich darin einig, dass der wirtschaftlichen Entwicklung in den beiden Ländern auch für ihre sicherheitspolitische Beziehung eine Schlüsselrolle zukommt. Besonders detailliert und aufschlussreich sind dabei die Daten und die auf ihnen beruhende Analyse von Makocki und Popescu in ihrem Kapitel „Drehpunkt Wirtschaft“. Sie stellen einleitend fest, dass sich die Regierungen in Moskau und Peking erst nach zehn langen Verhandlungsjahren und der Verhängung westlicher Sanktionen gegen Russland wegen der Annexion der Krim auf ein Gaslieferabkommen geeinigt hätten, das später als „das größte Gasabkommen der Welt" bezeichnet worden sei, als ein „Megadeal“, der eine Umgestaltung der globalen
Energiemärkte einläuten und eine geostrategische Partnerschaft zwischen zwei Großmächten besiegeln würde. Wichtiger noch, die Partnerschaft sei vom Kreml als praktikable Alternative zu den Beziehungen zum Westen dargestellt worden, die es ermögliche, das Sanktionsregime auszugleichen. Li Fenglin, ein ehemaliger chinesischer Botschafter in Moskau, den die Autoren zitieren, entwickelte sogar die kühne Vision, dass der Vertrag die Grundlage für eine „Gemeinschaft mit einem gemeinsamen Schicksal" schaffen würde.

Zweieinhalb Jahre nach dem Gas-Megadeal, so Makocki und Popescu, seien die chinesisch-russischen Wirtschaftsbeziehungen weit von der Verwirklichung derartiger Visionen entfernt. Der Handel zwischen den beiden Ländern sei um 30 Prozent geschrumpft, von 88 Milliarden USD im Jahr 2014 auf 64 Milliarden USD im Jahr 2015. Dies sei vor allem auf den Rückgang der Preise für fossile Brennstoffe und die Rezession in Russland zurückzuführen. Infolgedessen hätten sich auch die vorher schon bestehenden Asymmetrien verschärft: Während China im Außenhandel Russlands weiterhin den ersten Platz einnehme, rangiere Russland jetzt im chinesischen Außenhandel nur auf Platz 17. Es treffe zwar zu, dass sich die Direktinvestitionen (FDI) Chinas in Russland 2014 verdoppelt hätten; sie machten aber lediglich 5,6 Prozent der gesamten FDI in diesem Land aus. Trotz des Sanktionsregimes und des schwierigen politischen Umfelds sei Europa nach wie vor die wichtigste Quelle ausländischer Direktinvestitionen in Russland; im Jahr 2015 hätten europäische Projekte in diesem Land die Chinas um ein Zehnfaches überstiegen. Auch bei den Finanzbeziehungen sehe das Bild nicht viel anders aus: Die Erwartungen, dass China eine wichtige Kapitalquelle für russische Unternehmen werde, die vom Westen auf die schwarze Liste gesetzt worden seien, hätten zurückgefahren werden müssen. Dies werde sich wahrscheinlich nicht ändern, so die Autoren. Trotz der formellen Opposition Chinas gegen die Sanktionen hielten sich die Geschäftsbanken stillschweigend an sie, wahrscheinlich aus Angst, dass sie von den lukrativeren US-Finanzmärkten ausgeschlossen werden könnten.

Auch die Arbeiten von Yukon Huang, Senior Fellow bei der Carnegie-Stiftung, legen nahe, dass sich die chinesisch-russischen Asymmetrien noch verstärken könnten. In seinem Artikel in der New York Times, der im Wesentlichen eine Kurzfassung seines Buches Cracking the China Conundrum: Why Conventional Economic Wisdom Is Wrong darstellt, vertritt er die These, dass die chinesische Erfolgsgeschichte weitergehen werde. China sei nie eine „normale“ Volkswirtschaft gewesen und werde es auch weiterhin nicht sein. Es habe in den vergangenen vier Jahr- 
zehnten im Durchschnitt fast zehn Prozent Wachstumsraten erlebt, was weltweit einen Rekord darstelle. Es sei das erste Entwicklungsland, das sich zu einer Großmacht entwickelt habe. Was einige für Schwächen der chinesischen Wirtschaft hielten, seien eigentlich Stärken: Unausgewogenes Wachstum sei kein Anzeichen für drohende Risiken, sondern vielmehr Bestandteil erfolgreicher Industrialisierung. Die steigende Verschuldung sei ein Zeichen für eine Vertiefung der Finanzen und nicht für eine Verschwendung von Ausgaben. Korruption habe das Wachstum angespornt, nicht zum Stillstand gebracht. Die glorreichen Tage der chinesischen Wirtschaft mit zweistelligen Wachstumsraten mögen vielleicht vorbei sein, Wachstumsraten von sechs Prozent im nächsten Jahrzehnt seien aber durchaus möglich. In diesem Tempo werde sich die chinesische Volkswirtschaft bis zum Jahr 2030 verdoppeln und nach kaufkraftbereinigten Wechselkursen berechnet vermutlich die größte der Welt werden.

\section{Besprochene Literatur}

Michal Makocki and Nicu Popescu: China and Russia: An Eastern Partnership in the Making? European Institute for Security Studies (EUISS), Chaillot Paper No. 140, Dezember 2016 https://www.iss.europa.eu/content/china-and-russiaeastern-partnership-making

Marcin Kaczmarski: The Decreasing Asymmetry in Russia-Chinese Relations: The Consequences of the Western Policy Shift towards China, Finnish Institute of International Affairs (FIIA), 28. Februar 2018, https://www.fiia.fi/en/publication/thedecreasing-asymmetry-in-russia-china-relations

Paul Stronski und Nicole Ng: Cooperation and Competition: Russia and China in Central Asia, the Russian Far East, and the Arctic, Carnegie Endowment for International Peace, 28. Februar 2018, http://carnegieendowment.org/2018/02/28/ cooperation-and-competition-russia-and-china-in-central-asiarussian-far-east-and-arctic-pub-75673

Paul J. Kohlenberg und Nadine Godehardt: Chinas globale Konnektivitätspolitik: Zum selbstbewussten Umgang mit chinesischen Initiativen, Stiftung Wissenschaft und Politik (Berlin), SWPAktuell 2018/A 18, März 2018, https://www.swp-berlin.org/ fileadmin/contents/products/aktuell/2018A18_khb_gdh.pdf Yukon Huang: China's Economy Is Not Normal. It Doesn't Have to Be, The New York Times, 13. März 2018, https://www.nytimes. com/2018/03/13/opinion/china-economy-corruption.html 